\title{
Olivenöl-Schokolade als Herzschutz
}

Dunkle Schokolade mit Olivenöl angereichert könnte für Herz und Gefäße von Vorteil sein. Anhaltspunkte dafür fanden italienische Forscher um Dr. Rossella Di Stefano von der Universität in Pisa in einer kleinen randomisierten Studie.

30 gesunde Probanden, die mindestens drei kardiovaskuläre Risikofaktoren aufwiesen, bekamen über 28 Tage täglich $40 \mathrm{~g}$ dunkle Schokolade mit einem Kakaoanteil von $70 \%$ serviert, die entweder Olivenöl oder getrocknete rote Panaia-Äpfel (die besonders viele Polyphenole und Antioxidanzien enthalten sollen) enthielt. Nach 14 Tagen erfolgte ein Wechsel der Sorte.

\section{HDL-Cholesterin steigt}

Vier Wochen später zeigte sich, dass sich mit der mit Apfel angereicherten Schokolade - außer einem nicht signifikanten Rückgang der Triglyzeride - keine ausgeprägten Effekte herbeiführen ließen, was auch an der geringen Menge des Apfelzusatzes liegen könnte, so Di Stefano.

Die olivenölhaltige Variante führte dagegen zu einem signifikanten Anstieg von endothelialen Progenitorzellen (EPC) im Blut. EPC sorgen für Reparaturen an den Gefäßwänden und eine reibungslose Endothelialfunktion. Bei Patienten mit kardiovaskulären Risikofaktoren ist die Menge der zirkulierenden EPC reduziert. Zudem waren nach Genuss der OlivenölSchokolade ein signifikanter Anstieg des $\mathrm{HDL}$-Cholesterins und eine moderate Senkung des diastolischen Blutdrucks zu verzeichnen.

Täglich etwas dunkle Schokolade, die mit natürlichen Polyphenolen aus nativem Olivenöl angereichert sei, zu essen, könne langfristig positive Effekte auf das kardiovaskuläre Risiko haben, resümierte Di Stefano.

Den Sponsor der Studie, den toskanischen Hersteller dieser neuen Schokoladenvariante "Toscolata Olio" aus Arezzo, wird das freuen - denn eine Tafel kostet 11 Euro. Ob der lebenslängliche Verzehr von $40 \mathrm{~g}$ Schokolade nicht irgendwann auch negative metabolische Effekte haben könnte, wurde nicht

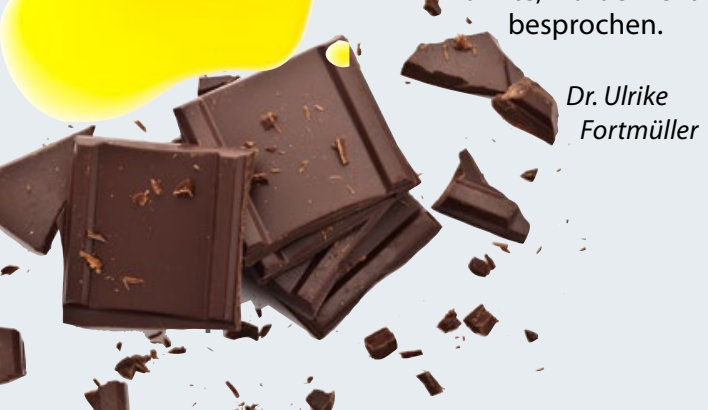

dern", wird der Koautor Prof. Johannes Brachmann vom Klinikum Coburg in einer Pressemitteilung der ESC zitiert.

\section{Evidenz gestärkt}

Bisher wurde die Katheterablation hauptsächlich bei Patienten mit Vorhofflimmern und normaler Herzfunktion angewandt. In den letzten Jahren deutete sich aber zunehmend an, dass die Intervention auch bei Patienten mit eingeschränkter linksventrikulärer Funktion eine hohe Erfolgsrate verspricht. So hatte die 2016 publizierte Studie von Di Biase et al. die gute Wirksamkeit der Intervention bei dieser schwer zu behandelnden Patientenpopulation erstmals an einer größeren Patientenzahl belegen können.

\section{Klinikeinweisungen wegen} Herzinsuffizienz reduziert

Die CASTLE-AF-Studie schreibt diese Erfolgsgeschichte nun fort. Prof. Nassir Marrouche von der Universität Utah Health in Salt Lake City hat die Ergebnisse auf einer ESC-Hotline-Session vorgestellt.

Nach einem mittleren Follow-up von 37,8 Monaten zeigte sich, dass die Sterblichkeit und die Rate an Klinikeinweisungen aufgrund einer sich verschlechternden Herzinsuffizienz - der primäre Endpunkt - durch eine Katheterablation um $38 \%$ deutlich im Vergleich zur Standardtherapie gesenkt wurden $(28,5$ vs. 44,6\%). Die Gesamtsterblichkeit ging um $47 \%$ zurück (13,4 vs. $25 \%)$, die kardiovaskuläre Mortalität um $51 \%$. Auch die Häufigkeit von kardiovaskulär bedingten Klinikeinweisungen nahm um $28 \% \mathrm{ab}$.

\section{Vorhofflimmern-Rezidive seltener}

Im Studienverlauf waren Vorhofflimmern-Rezidive bei den Patienten, die eine Katheterablation erhielten, seltener als in der Standardtherapie-Gruppe. Auch die linksventrikuläre Auswurffraktion verbesserte sich nach dem Eingriff deutlicher, nach 60 Monaten war der Unterschied statistisch signifikant.

Insgesamt wurde in der multizentrischen Studie bei 179 Patienten aufgrund von paroxysmalem oder persistierendem Vorhofflimmern eine Radiofrequenz-Ablation vorgenommen. 185 Patienten er-

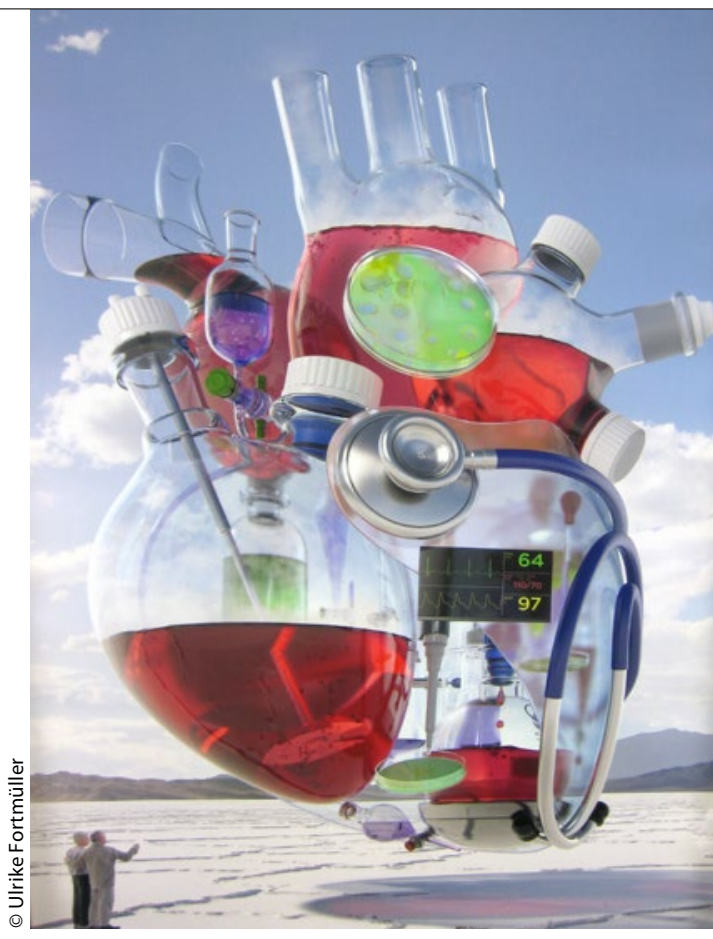

Herz in Not. Bei Herzschwäche und Vorhofflimmern wirkt die Katheterablation erstaunlich gut.

hielten eine nach den AHA/ACC- und ESC-Leitlinien von 2006 leitliniengerechte medikamentöse VorhofflimmernTherapie. Hierbei sollte, wenn möglich, ein Sinusrhythmus angestrebt werden.

\section{Mehrere Ablationen waren erlaubt}

Alle Patienten hatten eine linksventrikuläre Auswurffraktion von $\leq 35 \%$ und eine NYHA-Klasse $\geq 2$. Zu beachten ist auch, dass alle Teilnehmer bereits als Primär- oder Sekundärprävention einen ICD/CRT-D mit HomemonitoringFunktion implantiert bekommen hatten, was das Sterberisiko der Patienten in beiden Gruppen beeinflusst haben könnte. Einschlusskriterium war zudem das Versagen oder eine bestehende Intoleranz von mindestens einem Antiarrhythmikum oder die Ablehnung des Patienten, ein solches Medikament zu nehmen.

Die hohe Erfolgsrate der Katheterablation ist sicherlich auch dem Umstand $\mathrm{zu}$ verdanken, dass den behandelnden Kardiologen erlaubt war, weitere Ablationen vorzunehmen, wenn sie dies für nötig hielten.

Veronika Schlimpert

Quelle: Hot Line: Late-Breaking Clinical Trials 1 "Catheter ablation versus standard conventional treatment in patients with left ventricular dysfunction and atrial fibrillation: the CASTLE-AF trial", ESC-Kongress, 27.8.2017 in Barcelona 